\title{
Cirugía sin incisiones como tratamiento miniinvasivo del vólvulo sigmoideo a través del sitio de la colostomía. Reporte de caso
}

\author{
Juan Manuel Sotelo, Javier Oviedo, Romina Di Vietro, Rodrigo Moran Azzi \\ Sector Coloproctología, Sanatorio Güemes de Buenos Aires. CABA, Argentina.
}

\begin{abstract}
RESUMEN
El vólvulo sigmoideo (VS) es la forma de presentación más frecuente de los vólvulos colónicos, produciendo un abdomen agudo obstructivo, principalmente en pacientes añosos. La tomografía computada es considerada el gold standard para el diagnóstico. El tratamiento depende de varios factores, entre ellos, el estado general del paciente y la experiencia del cirujano actuante. El objetivo es presentar una forma miniinvasiva y sin cicatriz de resolver el VS no complicado en un paciente añoso.
\end{abstract}

Palabras claves: Vólvulo intestinal; Vólvulo sigmoideo; Procedimientos quirúrgicos; Sin incisión

\section{ABSTRACT}

Sigmoid volvulus (SV), the most common presentation of colonic volvulus, produces an acute obstructive abdomen mainly in elderly patients. Computed tomography is considered the gold standard for diagnosis. Treatment depends on several factors, including the general condition of the patient and the experience of the surgeon. The objective is to present a minimally invasive and incisionless approach to resolve an uncomplicated SV in an elderly patient.

Keywords: Sigmoid Volvulus; Surgical Procedures; Incisionless

\section{INTRODUCCIÓN}

El vólvulo colónico es una causa poco común de abdomen agudo obstructivo, representa el 2-3\% de las causas en EEUU, pero hasta un 10-50 \% en otras zonas del mundo, entre ellas América Latina.

El vólvulo sigmoideo es el más frecuente, representando entre un 50 a $90 \%$ de los casos. ${ }^{1}$

Los más afectados son los mayores de 70 años, institucionalizados, postrados, con hipotrofia muscular, constipación crónica y que muchas veces sufren de trastornos neurológicos o psicológicos. ${ }^{2}$

El método de diagnóstico considerado gold standard es la tomografía computada.

Múltiples son los tratamientos de los cuales disponemos para su resolución, podemos resumirlos en conductas endoscópicas, radiológicas y quirúrgicas. La devolvulación y descompresión endoscópica es el tratamiento de elección en pacientes compensados y sin signos clínicos ni radiológicos de isquemia o perforación, con un éxito aproximado de hasta un $90 \%$ en algunas series, asociado a la colocación de un tubo transanal. Sin embargo, se observa una alta tasa de recurrencia, por lo que muchos autores realizan una cirugía electiva días posteriores a la videocolonoscopía, luego de una mejoría del cuadro clínico y de prepara-

Los autores declaran ausencia de conflictos de interés.

Juan Manuel Sotelo

maquisotelo@gmail.com.ar

Recibido: agosto de 2020. Aceptado: octubre de 2020. ción intestinal. En pacientes con sospecha de perforación, isquemia del colon o en aquellos en quienes la endoscopia fue fallida, la resección quirúrgica es lo recomendado. La extensión de la misma; la vía de abordaje y la confección de una anastomosis primaria, con o sin derivación proximal, o de una colostomía definitiva, dependen del estado del paciente y de la experiencia del cirujano actuante. ${ }^{1,2}$

El objetivo es presentar un caso de VS en un paciente añoso, ASA III (American Society of Anesthesiologists) resuelto de forma miniinvasiva sin incisiones abdominales.

\section{CASO CLÍNICO}

Se presenta el caso de un paciente masculino de 93 años, con antecedentes de hipertensión arterial, hiperplasia prostática benigna, enfermedad de Parkinson, postración e hipotrofia muscular abdominal. Ingresa a la guardia por cuadro de distensión y dolor abdominal de $72 \mathrm{~h}$ de evolución, asociado a falta de eliminación de gases y catarsis negativa.

Al interrogatorio refiere episodios similares intermitentes en los últimos 5 años, que resolvían con maniobras de movilización y compresión abdominal por parte de acompañante y kinesiólogo en su domicilio.

Al examen físico se presenta compensado, abdomen distendido, asimétrico, con impronta intestinal en pared abdominal, dolor difuso, sin defensa ni reacción peritoneal. Al tacto rectal, ampolla distendida con restos de materia fecal. 


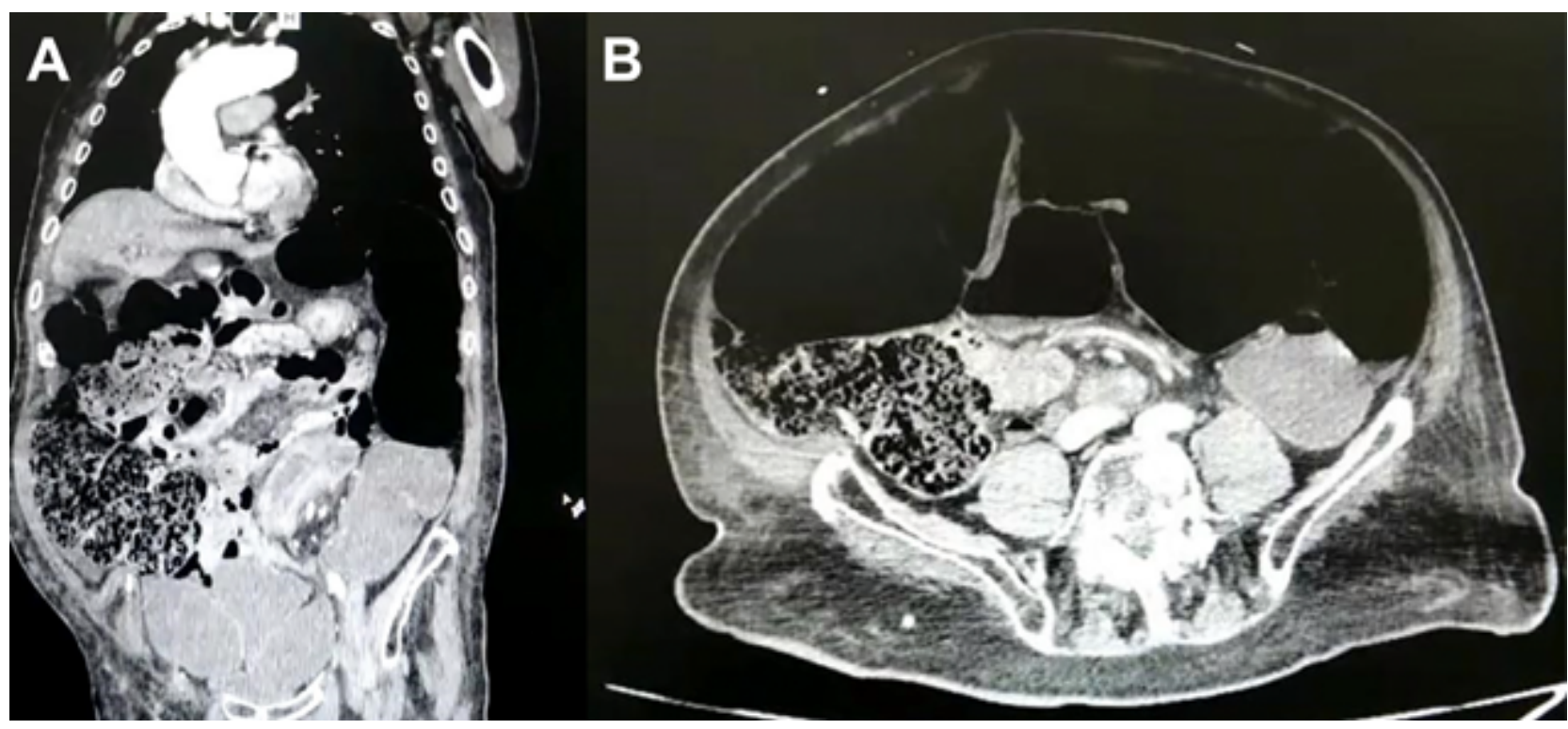

Figura 1: Pie de vólvulo (flecha). Distensión colónica.

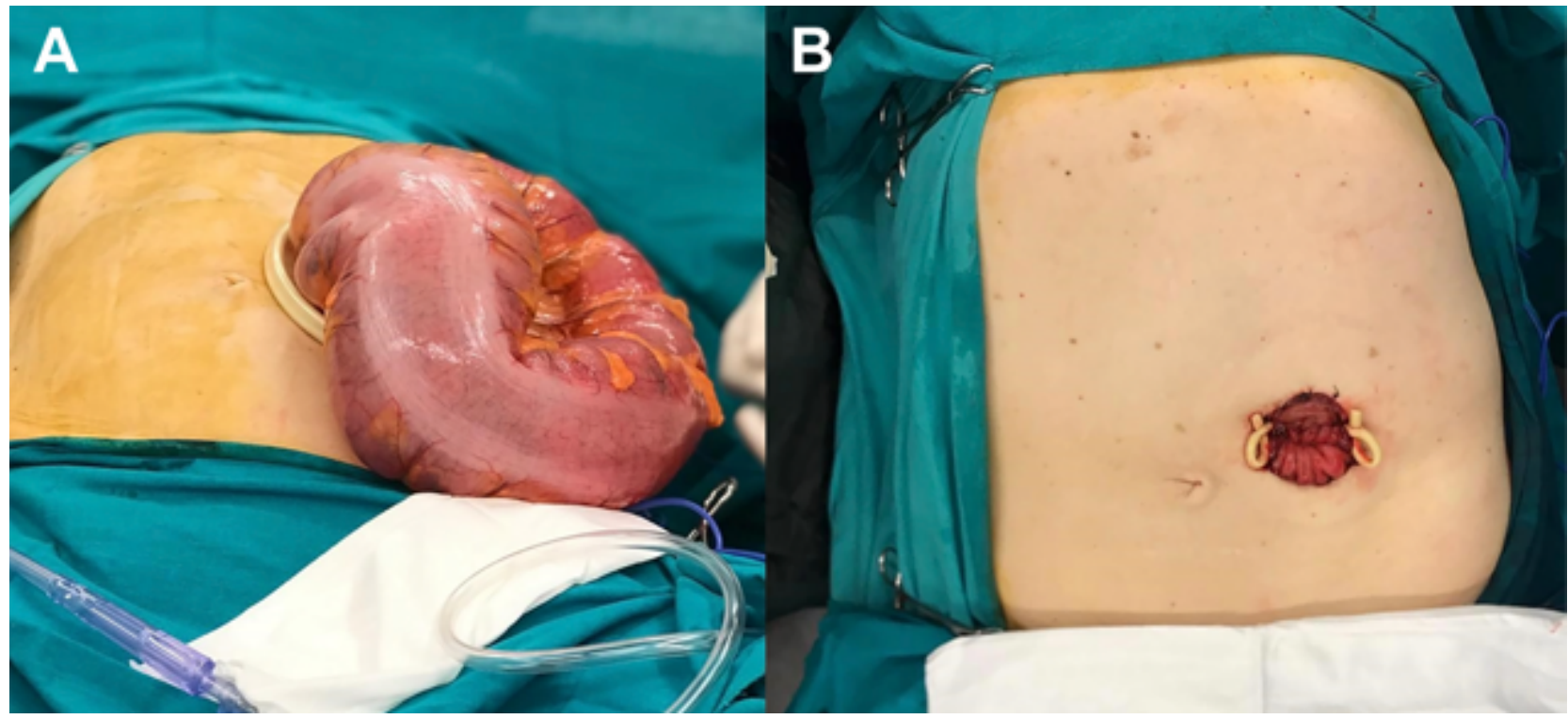

Figura 2: Vólvulo exteriorizado. Colostomía en caño de escopeta.

Se realiza tomografía computada de tórax, abdomen y pelvis con contraste endovenoso, donde se observa un segmento de colon sigmoideo con disminución del calibre, que se interpreta como pie de vólvulo, con gran distensión colónica retrógrada y abundante materia fecal (MF) en colon derecho (Figura 1).

Se decide VCC de urgencia, la cual progresa hasta $70 \mathrm{~cm}$ del margen anal, evidenciando zona volvulada que se logra franquear, drenando gran cantidad de aire y no pudiendo progresar por abundante MF. No se observan signos de isquemia o necrosis. Se coloca sonda transanal K227.

Evoluciona favorablemente, con tolerancia a la dieta, gases positivos y catarsis líquida por sonda, retirando la mis$\mathrm{ma}$ a las $48 \mathrm{~h}$. Posteriormente comienza con distensión abdominal progresiva, agregando dolor abdominal, por lo que se decide conducta quirúrgica el día 5.

Previa anestesia general e intubación orotraqueal se coloca al paciente en posición de Lloyd Davies modificada. Se realiza antisepsia e infiltración con anestesia local. Se realiza incisión circunferencial de $3 \mathrm{~cm}$ de diámetro aproximadamente, en el flanco izquierdo. Se incide aponeurosis longitudinalmente, se diseca con maniobras romas a través del músculo recto anterior del abdomen y se ingresa a cavidad. Se coloca retractor de piel tipo Alexis y se exterioriza colon sigmoideo redundante y volvulado, procediendo a devolvulación manual. Se realiza ligadura de mesocolon sigmoideo y sección colónica completando sigmoidectomía. Se confecciona colostomía según técnica de Mikulicz (Figura 2).

La duración aproximada del procedimiento fue de 50 
minutos, con recuperación anestésica favorable y retorno a sala general.

Se instauró dieta $6 \mathrm{~h}$ posteriores a la cirugía, con buena tolerancia. El primer día postoperatorio presentó colostomía vital y funcionante, otorgándose el alta sanatorial el día 2.

\section{DISCUSIÓN}

El vólvulo sigmoideo es un cuadro agudo que afecta principalmente a pacientes de edad avanzada, que habitualmente presentan múltiples comorbilidades asociadas, lo que se traduce en mayor morbilidad y mortalidad postoperatoria. ${ }^{1}$

Como mencionamos anteriormente el tratamiento de elección es el endoscópico, siempre que el estado clínico del paciente lo permita y no se halle isquemia intestinal en el mismo. Sin embargo, debido a la alta tasa de fracaso y recurrencia del vólvulo, el tratamiento quirúrgico es necesario, pudiéndose realizar días posteriores a la endoscopia, con preparación colónica previa., ${ }^{1,2}$

Distintas series como las de Niels van der Naald et al. y Seow-En y Seow-Choen realizaron abordajes mínimos similares, con anastomosis primarias y buenos resultados. ${ }^{3,4}$ Otros, como Alhindawi et al., realizaron procedimiento de Hartmann en pacientes añosos a través del sitio de la colostomía, sin presentar morbimortalidad perioperatoria y corta estadía hospitalaria. ${ }^{5}$ Sin embargo, estos estudios presentan algunas limitaciones, como tratarse de series de casos y estudios retrospectivos, al igual que la mayor parte de la bibliografía disponible en la actualidad.

Nuestro caso presenta un paciente añoso con importantes comorbilidades, ASA III, por lo que decidimos realizar esta secuencia de tratamiento, en primera instancia con el fin de evitar una cirugía. Debido a la evolución tórpida del paciente posterior al tratamiento endoscópico y la necesidad de una resolución quirúrgica, optamos por una resección y abocamiento en caño de escopeta, realizando el abordaje a través del sitio donde implantaríamos la colostomía, buscando y logrando de esta manera evitar otra incisión mayor, tiempos quirúrgicos prolongados, una posible dehiscencia anastomótica o de muñón rectal, con sus posteriores consecuencias, y obteniendo una rápida recuperación postoperatoria y egreso sanatorial.

Consideramos se trata de una técnica segura, efectiva y fácil de reproducir, tanto por cirujanos generales como por coloproctólogos, que evita complicaciones asociadas a una incisión mayor, anastomosis o muñón rectal, por lo que podría ser una opción muy útil para pacientes añosos con características clínicas desfavorables.

\section{REFERENCIAS}

1. Dolejs SC, Guzman MJ, Fajardo AD, Holcomb BK, Robb BW, Waters JA. Contemporary management of sigmoid volvulus. J Gastrointest Surg 2018; 22:1404-11.

2. Perrot L, Fohlen A, Alves A, Lubrano J. Management of the colonic volvulus in 2016. J Visc Surg 2016;153:183-92.

3. van der Naald N, Prins I, Otten K, Kumwenda D, Bleichrodt RP. Novel approach to treat uncomplicated sigmoid volvulus combining minimally invasive surgery with enhanced recovery, in a rural

hospital in Zambia. World J Surg 2018;42:1590-96.

4. Seow-En I, Seow-Choen F. Sigmoid volvulus treated by miniincision. Tech Coloproctol 2014;18:1169-71.

5. Alhindawi R, Kelly N, Holubar S. Incisionless Hartmann's procedure: an innovative minimal access technique for surgical treatment of sigmoid volvulus in debilitated patients with faecal incontinence. Tech Coloproctol 2008;12:337-39.

\section{COMENTARIO}

En el presente artículo los autores hacen un breve resumen del vólvulo sigmoideo y de sus opciones terapéuticas. Tal como ocurre en el caso clínico presentado, los pacientes con vólvulo sigmoideo suelen ser, en su mayoría, añosos, comórbidos y frágiles, lo que los hace más propensos a desarrollar complicaciones postoperatorias. Los métodos miniinvasivos son de elección en estos casos, dado que generan menor respuesta inflamatoria y menor dolor que la cirugía abierta, acelerando la recuperación luego del procedimiento.

Después de que el tratamiento endoscópico resultara insuficiente, los cirujanos del caso presentado optan por la cirugía resectiva. Para ello utilizan una innovadora técnica, escasamente descripta en la bibliografía. La misma se basa en la exteriorización del colon, la resección y la extracción de la pieza a través del sitio de maduración de la colostomía y la fístula mucosa. Esto resulta especialmente útil y adecuado en pacientes con vólvulo sigmoideo, debido a que suelen tener un dolicosigma, con mesos largos y laxos, lo que permite una adecuada movilización, sin necesidad de amplias laparotomías. La gran ventaja de esta técnica es que, debido a su pequeña incisión, se logran conservar los importantes beneficios de la miniinvasividad, especialmente valiosos en estos pacientes con alto riesgo quirúrgico.

Juan Pablo Campana Hospital Italiano. CABA, Argentina. 